\title{
Finite-size scaling of Polyakov's loop in the 2D Abelian Higgs model
}

\author{
Judah Unmuth-Yockey* \\ Syracuse University \\ E-mail: jfunmuthesyr.edu \\ Jin Zhang \\ University of California Riverside \\ E-mail: jzhan039@ucr.edu \\ Johannes Zeiher \\ Max-Planck-Institut für Quantenoptik \\ E-mail: johannes.zeiher@mpq.mpg.de
}

\author{
Alexei Bazavov \\ Michigan State University \\ E-mail: bazavov@msu.edu \\ Shan-Wen Tsai \\ University of California Riverside \\ E-mail: shan-wen.tsai@ucr.edu \\ Yannick Meurice \\ University of Iowa \\ E-mail: yannick-meurice@uiowa.edu
}

\begin{abstract}
Starting with the 2D Abelian Higgs model with the quartic self-coupling taken infinitely large we study the finite-size scaling of the Polyakov loop. We find an exponential decay for large temporal extents which is dictated by the energy gap between the ground states of a system with the Polyakov loop inserted, and one without. We study this system using the tensor renormalization group, and we take the continuous-time limit to obtain a quantum Hamiltonian where gauge invariance has been maintained exactly. Comparing with numerical results from the density matrix renormalization group we find universal features of the finite-size scaling of the energy gap survive this continuous-time limit. We propose an optical-lattice ladder to quantum simulate this model, and observe the universal features of the energy gap scaling.
\end{abstract}

The 36th Annual International Symposium on Lattice Field Theory - LATTICE2018

22-28 July, 2018

Michigan State University, East Lansing, Michigan, USA.

\footnotetext{
* Speaker.
} 


\section{Introduction}

Recently there has been a vigorous effort to quantum simulate lattice gauge models. This effort has been aimed at first-step models which are in low spatial dimensions and have only a few fields. A prime candidate is the Abelian Higgs model in 1+1 space-time dimensions and theoretical proposals are already being made [1, 2, 3]. In Refs. [4, 5] a method to quantum simulate the lattice Abelian Higgs model in 2D was proposed. In that proposal, we suggest to use discrete integer-valued fields and to maintain gauge invariance exactly from a Fourier expansion of compact variables leading to a change of variables where the partition function is written as a product of tensor contractions. Using this formulation we are able to measure the Polyakov loop, and we study its universal features. On the experimental side, we propose to use a physical ladder implemented in an optical lattice, with a single atomic species and a single particle per rung. In addition, we show that an even simpler model can be tested first using the same experimental technology, the quantum Ising model. In this case there are two legs in the ladder representing the two spin states.

\section{The model}

The lattice gauge theory we focus on is the Abelian Higgs model in two Euclidean dimensions,

$$
\begin{aligned}
S= & -\beta_{p l} \sum_{x} \sum_{v<\mu} \cos \left(A_{x, \mu}+A_{x+\mu, v}-A_{x+v, \mu}-A_{x, v}\right) \\
& -2 \kappa \sum_{x} \sum_{v=1}^{2} \cos \left(\theta_{x+v}-\theta_{x}+A_{x, v}\right) .
\end{aligned}
$$

Here the periodic gauge fields are $A_{x, \mu}$, and the periodic scalar fields are $\theta_{x}$. In this case the Higgs mode has been taken infinitely massive. The gauge coupling is $\beta_{p l}=1 / g^{2}$, and $\kappa$ is the coupling between the gauge field and the scalar field. This model is analogous to the Schwinger model, with the fermion field replaced by a complex scalar field. This model is believed to be confining, in that there is a linear potential between charges in the continuum limit. This is motivated by a study of the topological solutions of this model, which are responsible for confinement in the "Higgs" phase.

The partition function for this model before the change to integer fields is given by

$$
Z=\int \mathscr{D}\left[A_{x, \mu}\right] \mathscr{D}\left[\theta_{x}\right] e^{-S}
$$

where the integration over the compact field variables is $0 \leq A_{x, \mu}, \theta_{x} \leq 2 \pi$. To carry out the compact field integration we Fourier expand the Boltzmann weights,

$$
\begin{gathered}
e^{\beta_{p l} \cos \left(F_{x, \mu v}\right)}=\sum_{m=-\infty}^{\infty} I_{m}\left(\beta_{p l}\right) e^{i m F_{x, \mu v}} \\
e^{2 \kappa \cos \left(\theta_{x+v}-\theta_{x}+A_{x, v}\right)}=\sum_{n=-\infty}^{\infty} I_{n}(2 \kappa) e^{i n\left(\theta_{x+v}-\theta_{x}+A_{x, v}\right)} .
\end{gathered}
$$

This expansion factorizes the integration on the sites and links, and the final partition function is written in terms of the integer fields $n$, and $m$. There are constraints on the $n$ and $m$ variables from 


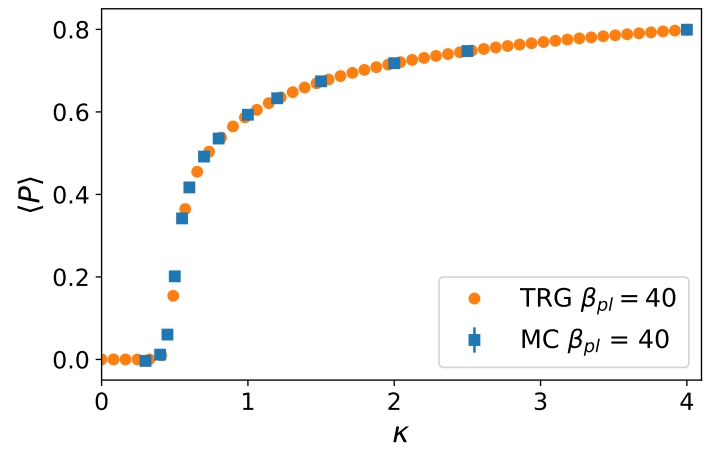

Figure 1: A comparison between calculations done of the Polyakov loop using the TRG and Monte Carlo. Here the calculations were done for a fixed gauge coupling, across a range of $\kappa$ values for a fixed space-time lattice of $16 \times 16$. The circles are TRG data, while the square markers are the Monte Carlo data.

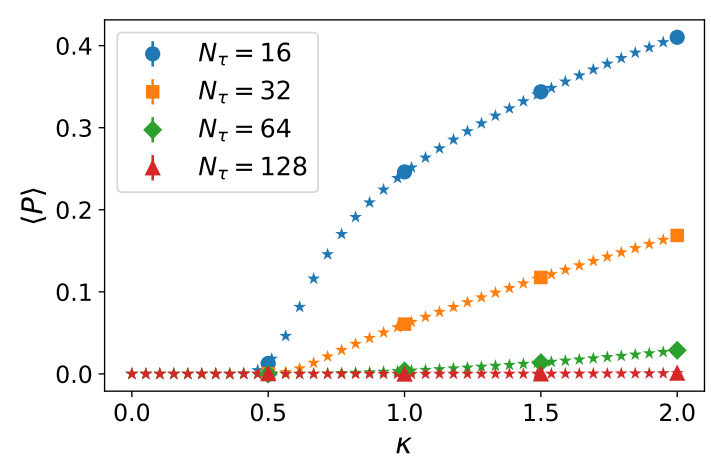

Figure 2: A comparison between the TRG and Monte Carlo at a fixed spatial volume, and fixed gauge coupling. Here the temporal extent of the lattice was varied. The little stars are the TRG data, while the larger markers are the Monte Carlo data.

the integration which can be solved immediately, giving a partition function

$$
Z=\sum_{\{m\}}\left(\prod_{x, \mu v} I_{m}\left(\beta_{p l}\right)\right)\left(\prod_{x, \mu} I_{m-m^{\prime}}(2 \kappa)\right) .
$$

This reformulation of the model is completely gauge-invariant; in fact, even if the infinite sum of the $m$ field is truncated, gauge invariance is maintained. Furthermore, we can rewrite this in terms of a local tensor and use the tensor renormalization group (TRG) to study the model [5].

\section{The Polyakov loop}

In the quantum simulation, one needs an observable to make a measurement and compare experiment with theory. In this case we considered the Polyakov loop and the free energy associated with the insertion of a static charge into the system. The Polyakov loop is a Wilson loop wrapped around the periodic temporal direction of the lattice.

$$
P=\prod_{n=1}^{N_{\tau}} U_{x^{*}+n \hat{\tau}, \tau}
$$

Here the product is over the gauge fields associated with the temporal links for a single spatial site. The expectation value of this operator is given by the usual expression,

$$
\begin{aligned}
\langle P\rangle & =\frac{1}{Z} \int \mathscr{D}\left[A_{x, \mu}\right] \mathscr{D}\left[\theta_{x}\right] e^{-S} P \\
& =\sum_{\{m\}}\left(\prod_{x, \mu v} I_{m}\left(\beta_{p l}\right)\right)\left(\prod_{x, \mu} I_{m-m^{\prime}}(2 \kappa)\right)\left(\prod_{n=1}^{N_{\tau}} \frac{I_{m-m^{\prime}-1}(2 \kappa)}{I_{m-m^{\prime}}(2 \kappa)}\right) .
\end{aligned}
$$




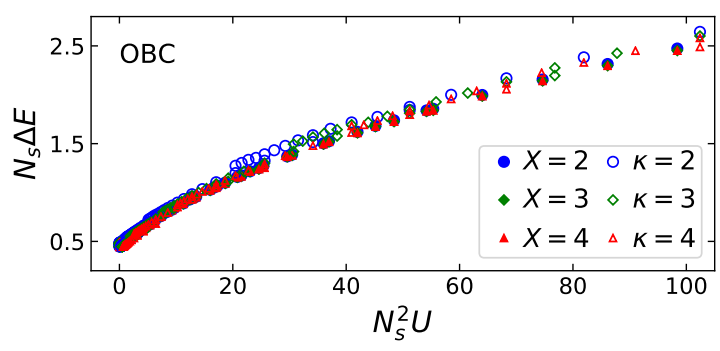

Figure 3: The data collapse for the energy gap associated with the Polyakov loop inserted into a system with open boundary conditions. Here there is data from both the fully discrete lattice model and the continuous-time limit for a few values of the spin-flip coupling, and the scalar-gauge coupling. As defined in the text, $U=g^{2} / a$

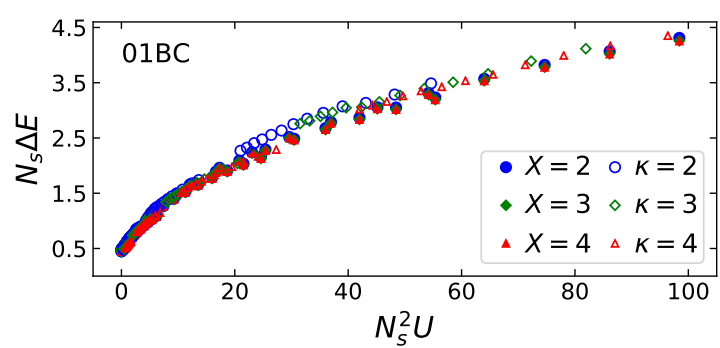

Figure 4: The data collapse for the energy gap between a system with open boundary conditions, and a system with an external field. There is no Polyakov loop in this scenario. This energy gap can be related to the energy gap from a Polyakov loop, and this is elaborated on in Refs. [4, 5].

In the second equality, we have carried out the Fourier expansion described in Sec. 2 and integrated out the gauge fields and scalar fields as before. This gives a representation of the Polyakov loop in terms of the integer $m$ fields. This expectation value can be studied using a tensor formulation as well.

\section{Comparison between Monte Carlo and TRG}

In order to verify the correctness of the tensor formulation for this model, and the tensor formulation of the Polyakov loop, we compared calculations with Monte Carlo simulations done using the original field variables. An example of a comparison can be seen in Fig. 1, where the Polyakov loop is compared for a fixed value of $\beta_{p l}$ across a range of $\kappa$ values at fixed space-time volume. In Fig. 2 we compared calculations of the Polyakov loop while varying the temporal extent, $N_{\tau}$, of the lattice. We find good agreement between the two methods.

The change observed in Fig. 2 led us to investigate the temporal extent, $N_{\tau}$, dependence of the Polyakov loop. We found that for sufficiently large $\kappa$ and $N_{\tau}$, the Polyakov loop scales like

$$
\langle P\rangle \simeq e^{-(\Delta E) a N_{\tau}} .
$$

This isn't completely surprising since it's known that the Polyakov loop is related to the free energy of a static charge by

$$
\langle P\rangle \simeq e^{-\Delta E / T}
$$

with $T$ the temperature. A well known result from thermal field theory is that $a N_{\tau}=\frac{1}{T}$, with $a$ the temporal lattice spacing. Here $\Delta E$ is the energy gap between a system with a Polyakov loop (an infinitely massive static charge inserted), and one without. We further investigated the finite-size scaling of the energy gap and the dependence on $\beta_{p l}$ and $\kappa$. In order to confirm the scaling we saw using the TRG, we compared with calculations of the energy gap using Monte Carlo [5].

By plotting $\Delta E$ for different spatial volumes across a range of $\beta_{p l}$ values, the data appear to exhibit $N_{s}$ dependence. In fact, by a change of variables one can rescale the energy gap to $N_{s} \Delta E$ 
and the gauge coupling to $N_{s}^{2} / \beta_{p l}=N_{s}^{2} g^{2}=N_{s}^{2} U$ to obtain a data collapse. This can be seen in Figs. 3 and 4 for a few $\kappa$ values. This collapse is only possible for $\kappa$ sufficiently large, and in a regime where $g^{2} N_{s}^{2}$ is not too large. Outside of this region the collapse breaks down. This is further explained in Ref. [5].

\section{Continuous time limit}

In order to make contact with reality in which time is continuous, the lattice model must be modified so that time is no longer discrete. This was worked out in detail in Refs. $[6,7,4,5]$. In our model this consists of taking the temporal couplings very large, that is, $\beta_{p l}, \kappa_{\tau} \rightarrow \infty$, while simultaneously taking the spatial couplings and temporal lattice spacing very small, $\kappa_{s}, a \rightarrow 0$. By taking this limit, but keeping combinations of these variables constant,

$$
U \equiv \frac{1}{\beta_{p l} a}=\frac{g^{2}}{a}, \quad Y \equiv \frac{1}{2 \kappa_{\tau} a}, \quad X \equiv \frac{2 \kappa_{s}}{a},
$$

one is, in essence, expanding the transfer matrix of the model about the identity,

$$
\mathbb{T} \simeq \mathbf{1}-a H+\ldots
$$

From this limit one can extract the Hamiltonian, $H$, of the model and for this case we find,

$$
H=\frac{U}{2} \sum_{i=1}^{N_{s}}\left(L_{i}^{z}\right)^{2}+\frac{Y}{2} \sum_{i}^{\prime}\left(L_{i+1}^{z}-L_{i}^{z}\right)^{2}-X \sum_{i=1}^{N_{s}} U_{i}^{x}
$$

with

$$
L^{z}|m\rangle=m|m\rangle, \quad U^{x}=\frac{1}{2}\left(U^{+}+U^{-}\right), \quad U^{ \pm}|m\rangle=|m \pm 1\rangle .
$$

Here $L^{z}$ is acting in the usual way for the $z$-component of angular momentum, and $U^{ \pm}$are analogous to raising and lowering operators; however, they are distinct from the raising and lowering operators of the angular momentum algebra. The special summation, $\Sigma^{\prime}$ is used to denote that the open boundary conditions must be taken into account.

Since the Polyakov loop has a representation in terms of the $m$ fields, it's natural to ask what is the continuous-time limit of that operator. Using the same limits from before we find the Polyakov loop adds an additional single-site term to the quantum Hamiltonian,

$$
P=\prod_{n=1}^{N_{\tau}} \frac{I_{m-m^{\prime}-1}(2 \kappa)}{I_{m-m^{\prime}}(2 \kappa)} \mapsto-\frac{Y}{2}\left(2\left(L_{i^{*}+1}^{z}-L_{i^{*}}^{z}\right)-1\right),
$$

changing the Hamiltonian to,

$$
\tilde{H}=H-\frac{Y}{2}\left(2\left(L_{i^{*}+1}^{z}-L_{i^{*}}^{z}\right)-1\right) .
$$

Here $H$ is the Hamiltonian of the Abelian Higgs model without the Polyakov loop from Eq. 5.3, and $i^{*}$ is the special site where the charge is inserted. In calculations this site was in the middle of the spatial lattice. From Eq. 5.6 it's clear that $\Delta E$ is the difference in the ground state energies of the two Hamiltonians. 
Knowing the quantum Hamiltonian for the Abelian Higgs model and the Hamiltonian for the Abelian Higgs model with a Polyakov loop inserted, we can calculate $\Delta E$ directly. The insertion of the Polyakov loop can be related to the presence of an external electric field. This is shown in Ref. [5]. Here we quote results from that study. Using the density matrix renormalization group we calculate the ground-state energies for the two Hamiltonians and compute their difference across the same range of gauge couplings and spatials sizes that was done in the fully discrete theory. In Figs. 3 and 4 both the data collapse from the discrete lattice theory, and the data collapse from the continuous-time limit are plotted. Here there are two energy gaps plotted, each showing it's respective collapse: the energy gap between a system with a Polyakov loop, and one without, $\Delta E=E_{\mathrm{PL}}^{(0)}-E^{(0)}$, and a system with an external field, and one without, $\Delta E=E_{01 \mathrm{BC}}^{(0)}-E^{(0)}$. This data collapse demonstrates that for sufficiently small $N_{s}^{2} g^{2}, N_{s} \Delta E=f\left(g^{2} N_{s}^{2}\right)$ is some function only of the combination $N_{s}^{2} g^{2}$, and furthermore that the collapse survives the continuous-time limit. In addition, the collapse is across values of the spin-flip coupling, $X$, as well as the gauge-scalar coupling, $\kappa$, from the original discrete lattice model.

\section{Ladder system Hamiltonian}

With the quantum Hamiltonian for the Abelian Higgs model, one needs a suitable optical lattice Hamiltonian with which to simulate the model. Here we propose a physical multi-leg ladder optical lattice to represent both the spatial lattice, and the angular momentum state-space. The Hamiltonian we propose is,

$$
\hat{H}=-\frac{J}{2} \sum_{i=1}^{N_{s}} \sum_{m=-s}^{s-1}\left(\hat{a}_{m, i}^{\dagger} \hat{a}_{m+1, i}+\text { h.c. }\right)-\sum_{i=1}^{N_{s}} \sum_{m=-s}^{s} \varepsilon_{m, i} \hat{n}_{m, i}+\sum_{i, i^{\prime}=1}^{N_{s}} \sum_{m, m^{\prime}=-s}^{s} V_{m, m^{\prime}, i, i^{\prime}} \hat{n}_{m, i}, \hat{n}_{m^{\prime}, i^{\prime}} .
$$

In this Hamiltonian, $|s|$ signifies the largest possible angular momentum state accessible for the system. The operators have the usual interpretation: $\hat{n}_{m, i}$ is a bosonic number operator at site $i$ counting state $m$, and $\hat{a}_{m, i}$ is the annihilation operator at site $i$ for state $m$. The couplings $J, \varepsilon$, and $V$ control the hopping between different states, the on-site potentials, and the nearest-neighbor interactions respectively. For an illustration of a five-state system see Ref. [4].

Of the terms in the ladder Hamiltonian, the nearest-neighbor interaction term is the most difficult to engineer experimentally. This is because of the quadratic nature of the potential specified by the Abelian Higgs Hamiltonian. In order to match the Abelian Higgs model, the potential must have the form,

$$
\begin{aligned}
V_{m, m^{\prime}, i, i^{\prime}} & =V_{m, m^{\prime}} \delta_{i^{\prime}, i+1} \\
& =\left(-\left|V_{0}\right|+\frac{Y}{2}\left(m-m^{\prime}\right)^{2}\right) \delta_{i^{\prime}, i+1} .
\end{aligned}
$$

This type of interaction can be achieved for an asymmetric lattice using a Rydberg-dressed potential. That is, the optical lattice spacing in the spatial dimension and the angular momentum dimension must be different. In terms of the leg and rung lattice spacing, this means $a_{l} \gg a_{r}$. By finding a specific tuning of $a_{l} / a_{r}$ one can achieve a quadratic potential for nearest neighbors in the angular momentum state-space. A quantitative analysis demonstrating the quadratic dependence can be found in Ref. [4]. 


\section{Conclusion}

We presented results from recent work in Refs. [4, 5] pertaining to the quantum simulation of the Abelian Higgs model and measuring universal features of the Polyakov loop within that model. We found that the energy gap associated with the Polyakov loop obeys a universal finite-size scaling in the discrete lattice model. The Abelian Higgs model has a simple continuous-time limit as a rotor model which is gauge-invariant. Moreover the Polyakov loop has a continuous-time limit, and the energy gap associated with it also shows a universal finite-size scaling in this continuous-time limit. We propose a physical multi-leg optical lattice ladder to simulate the Abelian Higgs model. The interactions on this optical lattice are all local, and the nearest neighbor interactions can be achieved using an asymmetric optical lattice with a Rydberg-dressed potential. Using the same experimental technology and set-up, the quantum Ising model could be simulated as a first step in understanding the systematics of this optical lattice ladder simulator.

Acknowledgments: SWT would like to thank the National Science Foundation for work supported under Grant No. DMR-1411345. YM would like to thank the U.S. Department of Energy for work supported under Award Number DE-SC0010113. JU would like to thank the U.S. Department of Energy for work supported under Award Number DE-SC0009998.

\section{References}

[1] Y. Kuno, K. Kasamatsu, Y. Takahashi, I. Ichinose, and T. Matsui. "Real-time dynamics and proposal for feasible experiments of lattice gauge-Higgs model simulated by cold atoms”. New J. Phys. 17, 063005 (2015).

[2] Y. Kuno, S. Sakane, K. Kasamatsu, I. Ichinose, and T. Matsui. "Quantum simulation of (1+1)-dimensional U(1) gauge-Higgs model on a lattice by cold Bose gases”. Phys. Rev. D 95, 094507 (2017).

[3] D. Gonzalez-Cuadra, E. Zohar, and J. I. Cirac. "Quantum simulation of the Abelian-Higgs lattice gauge theory with ultracold atoms”. New J. Phys. 19, 063038 (2017).

[4] Jin Zhang, J. Unmuth-Yockey, J. Zeiher, A. Bazavov, S.-W. Tsai, Y. Meurice. "Quantum simulation of the universal features of the Polyakov loop". arXiv:1807.09186 [hep-lat] (2018).

[5] Judah Unmuth-Yockey, Jin Zhang, Alexei Bazavov, Yannick Meurice, Shan-Wen Tsai. "Universal features of the Abelian Polyakov loop in 1+1 dimension". arXiv:1803.11166 [hep-lat] (2018).

[6] John Kogut and Leonard Susskind. "Hamiltonian formulation of Wilson's lattice gauge theories". Phys. Rev. D 11, 395 (1975).

[7] Eduardo Fradkin and Leonard Susskind. "Order and disorder in gauge systems and magnets". Phys. Rev. D 17, 2637 (1978). 